\title{
Hatch Textures for Virtual Endoscopy
}

\author{
C. Schaller ${ }^{1}$, D. Wellein ${ }^{2}$, S. Born ${ }^{2}$, D. Bartz ${ }^{2}$ \\ ${ }^{1}$ WSI/GRIS, University of Tübingen \\ ${ }^{2}$ Visual Computing for Medicine, ICCAS, University of Leipzig \\ daniela.wellein@iccas.de
}

\begin{abstract}
We present an illustrative rendering extension for virtual endoscopy to enhance depth perception. For this purpose different hatch textures are applied and their ability to enhance 3D shape estimation is discussed. The stroke directions of the textures are aligned to the principal curvature directions of the surface. Starting with two patterns suggested by Kim et al. [1], we developed texture patterns for the use in our virtual endoscopy system. Altogether five different hatch textures are integrated within the framework and OpenGL-generated mipmaps are applied to account for aliasing effects on the textured surfaces.
\end{abstract}

\section{Introduction}

Virtual endoscopy can provide a fast non-invasive exploration method suited for screening, diagnosis, access planning and post-operative evaluation, depending on the clinical application area. While only minimal patient preparation is needed, it even can allow virtual access to obstructed or otherwise physically unreachable regions. Clinical application areas are amongst others virtual bronchoscopy, colonoscopy, and ventriculoscopy. A general overview can be found in [2]. Our framework for virtual endoscopy supports multiple applications such as cardio-vascular [3] or neuroendoscopic [4] exploration. It allows interactive and
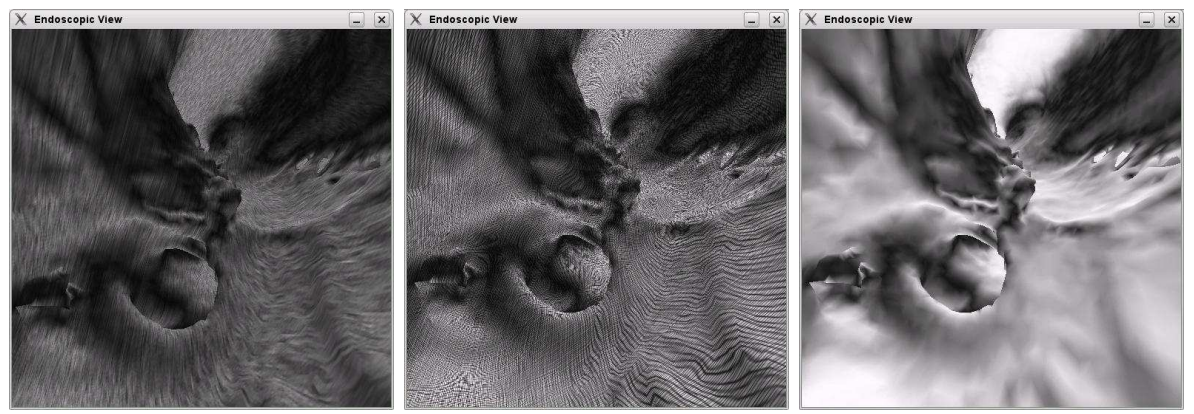

Fig. 1. Screenshot of our virtual endoscopy framework. Scene with texture a (left), with texture b (middle) and, with no texture (right). For the textures see Fig. 2 
intuitive handling on a common PC by combining polygonal surface rendering with a guided-navigation paradigm.

In virtual endoscopy, crucial depth information is significantly reduced by projecting a 3D scene onto a $2 \mathrm{D}$ screen. One of the main ideas of illustrative rendering is to provide additional visual cues for the interpretation of a scene. The most important source of depth cues in virtual endoscopy is the relative movement of objects due to shifts in perspective (motion parallax). Appropriate texturing, like with hatch textures, can provide additional depth cues and therefore enhances the perception of 3D shapes.

Ritter et. al [5] describe a hatching algorithm along with shadow-like depth indicators, that is used to increase depth perception for vascular structures. This visualization technique is validated by a large scale user study. Zander et al. [6] direct hatching strokes along the surface curvature. Their approach may be suitable for medical scenarios in general but proves to be problematic for realtime applications. Kim et al. demonstrate in [1] that hatch textures following one or two principal curvature directions of an $3 \mathrm{D}$ object facilitate the perception of 3D object geometry.

The remainder of this paper is structured as follows. In Section 2 we will introduce the textures utilized within our framework and their applicability to virtual endoscopy. Section 3 presents our results and in Section 4 we will discuss these results and give a brief outlook.

\section{Material and Methods}

All applied textures are depicted in Figure 2. In [1] the authors compared the subjects' ability to correctly estimate surface orientation of bumps on a landscape in four different conditions (no texture and three different textures). The textures a and c (Fig. 2) lead to the most accurate estimation of 3D objects and therefore will be used for texturing in our virtual environment system. Texture mapping is done with standard openGL functions. In openGL the textures are aligned orthogonal to the surface normals. This automatically leads to an alignment of the hatches along the curvature of the object surfaces.

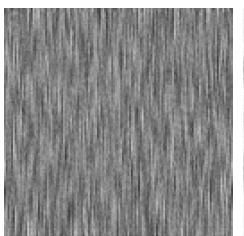

(a) a

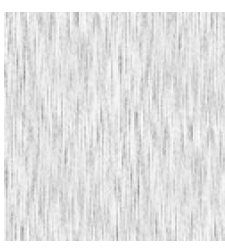

(b) b

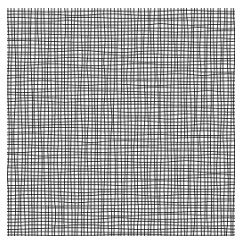

(c) c

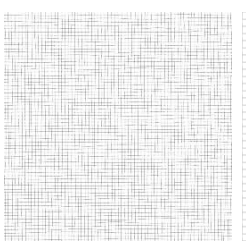

(d) $\mathrm{d}$

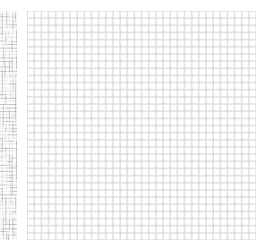

(e) e

Fig. 2. Textures. a) lic-like, one principal curvature direction b) contrast-reduced version of a c) grid, two principal curvature directions d) contrast-reduced version of c e) diamond pattern, principal curvature directions 
Due to the high contrast of both textures (a,c) the endoscopic scene is significantly darkened (Fig. 2). When these textures are mapped on distant structures the resulting aliasing-effects are quite strong. This produces an especially interfering flickering effect when navigating within the endoscopic view. Therefore, we adapted the original textures by lowering contrast and luminance, obtaining textures $\mathrm{b}$ and $\mathrm{d}$. While aliasing is reduced when applying the low-contrast textures b and d, nevertheless it is still perceivable. To account for this, we applied OpenGL-generated mipmaps. A mipmap is a downscaled versions of the original texture with reduced level of detail. The size of the object on the screen determines which downscaled version of the texture map is used.

We additionally created a fifth texture, the rectilinear diamond texture (Fig. $2 \mathrm{e})$. Like texture $\mathrm{c}$ and $\mathrm{d}$ it is bi-directional, which is - according to Kim et al. [1] - slightly superior to uni-directional hatch textures (a, b). The regularity of the strokes and its low contrast draws less visual attention and is therefore more suitable for virtual endoscopy than the textures a and $\mathrm{c}$ and their derivatives $\mathrm{b}$ and $\mathrm{d}$.

without mipmapping
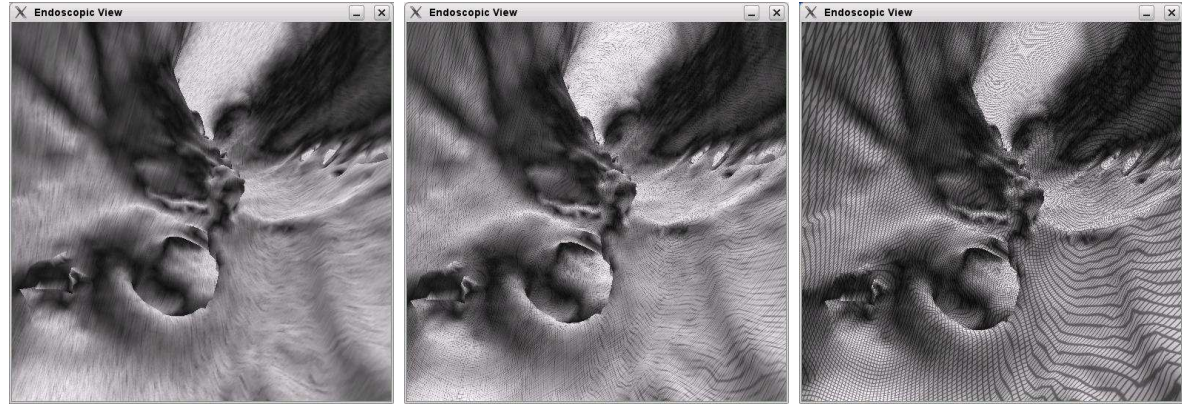

with mipmapping
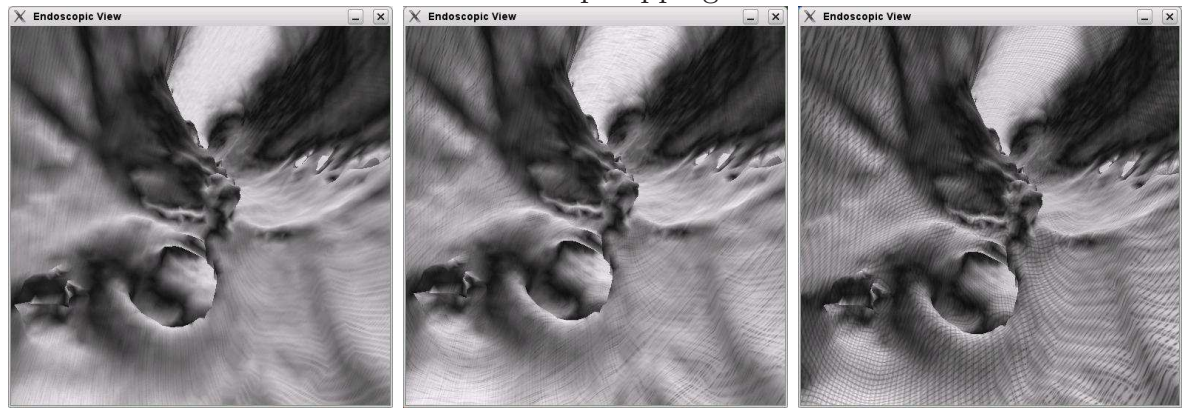

Fig. 3. The application of different textures (Fig. 2 b, d and e), with and without mipmapping. Texture b (left), texture d (middle) and, texture e (right) 


\section{Results}

Figure 1 displays the application of the original textures to our virtual endoscopy framework together with a non-textured scene. High-contrast textures result in a relatively dark endoscopic view (Fig. 1 left, middle) and aliasing leads to a flickereffect on distant objects while navigating through the scene. Using the contrastreduced textures the scenes are lightened up (Fig. 3). Simultaneously the aliasing effects, while still perceptible, are considerably reduced. The contrast-reduced textures permit to blend texture and surface, rather than completely replacing it. This allows for additional accuracy due to the underlying lighting. The OpenGLintrinsic mipmapping turns out to be a mixed blessing. While aliasing effects are significantly reduced, the down-scaled mipmaps produce additional artifacts due to discontinuities between different resolutions at texture patch boundaries (see Fig. 4).

\section{Discussion}

We extended our framework for virtual endoscopy by hatching. The applied textures were chosen because they facilitate 3D shape perception. Luminance and stroke thickness have a huge influence on the quality of the visualization. Hence, textures b and d result in lighter representations than textures a and c, whereas texture e offers a smoother and more regular appearance. In summary, hatch textured surfaces bear an advantage over non-textured surfaces in 3D shape estimation, but have to be carefully selected and at present manually adapted for any given data set.

The attainment of an optimal texture depends largely on the artifacts that need to be accounted for. Aliasing effects, like described above, can be mostly eliminated by using mipmaps, but this technique in turn causes artifacts of its own. First, in some scenarios the boundaries between different texture maps
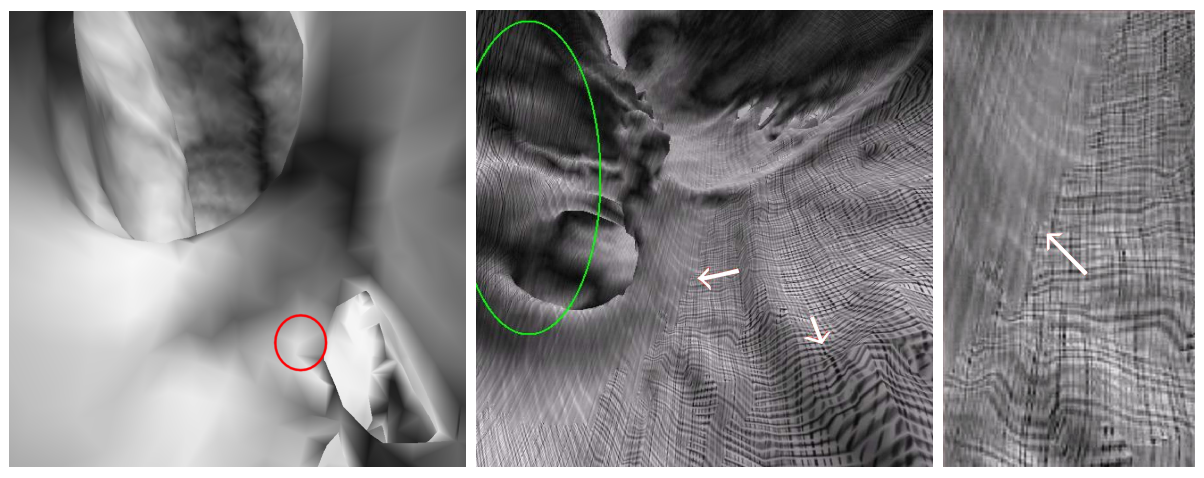

Fig. 4. Visualization artifacts. Diamond artifact (circle, left), blurred textures at an acute angle (ellipse, middle) visible texture boundaries (arrow, middle and right) 
are visible (Fig. 4 right, circle). Second, when using OpenGL-generated texture maps, boundaries between texture patches of differing level of detail become visible and, within the texture patch itself the strokes become discontinuous for the smaller texture maps. Smooth transitions between texture patches require manual adaptation. Weighting of the texture maps by contrast can account for smoother boundaries between different mipmap resolutions. A general problem is that OpenGL does not allow for anisotropic filtering. This leads to a blurring of textured surfaces when observed from an acute angle (Fig. 4 middle, green ellipse). A general advantage of texturing is, that diamond artifacts (Fig. 4 left, red circle) caused by insufficient triangle subdivision during 3D model generation is masked.

The next steps will include the weighting the textures according to vertex lighting and the integration of tonal art maps (TAM) like proposed by Praun et al. [7]. We also plan a psychophysical user-study to more accurately specify the benefits of hatch textures for virtual endoscopy.

\section{References}

1. Kim S, Hagh-Shenas H, Interrante V. Showing shape with texture: Two directions seem better than One. Proc HVEI. 2003;5007:332-339.

2. Bartz D. Virtual endoscopy in research and clinical practice. Comput Grap Forum. $2005 ; 24(1): 111-26$.

3. Bartz D, Gürvit O, Lanzendörfer M, et al. Virtual endoscopy for cardio vascular exploration. Proc CARS. 2001; p. 960-4.

4. Bartz D, Straßer W, Gürvit O, et al. Interactive and multi-modal visualization for neuroendoscopic interventions. Proc Eurographics Symp Data Vis. 2001; p. 157-64.

5. Ritter F, Hansen C, Dicken V, et al. Real-time illustration of vascular structures. Proc IEEE Vis. 2006; p. 877-84.

6. Zander J, Isenberg T, Schlechtweg S, et al. High quality hatching. Comp Graph Forum. 2004;23(3):421-30.

7. Praun E, Hoppe H, Webb M, et al. Real-time hatching. Proc ACM SIGGRAPH. 2001; p. 579-84. 